\title{
Division of labour in microorganisms: an evolutionary perspective
}

\author{
Stuart A. West \& Guy A. Cooper \\ Department of Zoology, University of Oxford, Oxford, OX1 3PS, UK \\ Stuart.West@zoo.ox.ac.uk
}

The division of labour, where individuals within a group specialise in certain tasks, has long been appreciated to be central to the evolution of complex biological societies. In recent years, a number of examples of division of labour in microorganisms have arisen, suggesting that this strategy may also be important within microbial species. In this Perspective, we proposed a set of conditions that define division of labour, and discuss recent examples according to these conditions. Furthermore, we discuss how clarifying what constitutes division of labour highlights key evolutionary question, including what form division of labour takes and why it is favoured by natural selection.

\section{Introduction}

Microbial cells within a population often show extreme variation in phenotype, which can arise via multiple mechanisms (Box 1). For example, when Escherichia coli cells are growing in a batch culture, a fraction of cells are in a transient non-growing state, termed persister cells, while the rest of the cells are growing normally ${ }^{1}$. A standard explanation for this phenotypic variation is that it represents a bet-hedging strategy, by which different phenotypes do better in different environments, and so the fitness of cells is increased by the production of variable phenotypes ${ }^{2,3}$. In the case of $E$. coli, the persister phenotype is beneficial because it enables survival under conditions of environmental stress, such as in the presence of antibiotics ${ }^{4}$.

However, many examples of phenotypic variation cannot be explained as bethedging. Instead, these other examples appear to involve some cells specialising to perform cooperative tasks that provide a benefit to other cells (FIG. 1A). For example, in Bacillus subtilis populations at stationary phase in liquid medium, some cells produce and excrete proteases to degrade proteins in the environment into smaller peptides, that can be used as nutrient sources ${ }^{5}$. As both these proteases and their degradation products diffuse freely, the production of proteases provides a benefit to the local population of cells, and not just those that produced them. We suggest that cooperation between different phenotypes is a defining feature of division of labour, and so these examples of phenotypic variation represent division of labour ${ }^{3,6,7}$.

Determining whether examples of phenotypic variation represent division of labour raises a range of new questions. Why would natural selection favour division of labour? Why would it be beneficial to have just a fraction of cells performing a trait, such as protease production, at a relatively high rate, rather than having all the cells performing that trait at a lower rate? If the division of labour is cooperative, why can't lineages that produce a lower proportion of the cooperative phenotype (cheats) ${ }^{8}$, invade the population, leading eventually to the loss of division of labour? Why is division favoured by natural selection in certain environments, for certain tasks, but not others? Or why should different mechanisms, such as phenotypic noise or environmental cues ${ }^{2}$ (Box 1), be used to produce phenotypic variation in different situations?

An understanding of division of can also elucidate the evolution of other biological processes, including virulence and multicellularity. The division of labour 
appears to be central to the success and virulence of pathogenic species, such as Cryptococcus gattii, the cause of fungal meningitis. During infection with $C$. gattii, the host immune response triggers a fraction of cells to develop tubular mitochondria, which somehow protect the cells with normal mitochondria from the host immune system $^{9}$. Furthermore, the discovery of division of labour in microorganisms suggests that broad comparisons could be made for why division of labour is favoured, across all levels of biological organisation, including human societies ${ }^{10}$. Finally, the division of labour between cells has played a crucial role in the evolution of multicellularity, so in order to understand how complex life on earth evolved, we need to understand why division of labour is favoured in microorganisms ${ }^{10-12}$.

In this Perspective, we clarify what constitutes division of labour within a microbial species. We propose a set of conditions that define division of labour and discuss whether a number of previously described examples of phenotypic plasticity represent adaptive division of labour. A precise definition matters, because imprecise definitions and ambiguity can obscure the fundamental problems and impede conceptual unification ${ }^{13,14}$. We outline key questions in the study of division of labour in microorganisms, focusing on what division of labour is, why it is favoured by natural selection, and what forms it can take.

\section{What is division of labour?}

We define the division of labour as when cooperating individuals specialise to perform specific tasks. This requires three conditions: (1) individuals perform different tasks (phenotypic variation); (2) some individuals perform cooperative tasks that benefit other individuals (cooperation); (3) this dividing of tasks provides an inclusive fitness benefit to all the individuals involved (adaptation).

A behaviour or trait represents cooperation if it benefits another individual, and has been selected for, at least partially, because of this benefit ${ }^{14}$. Cooperation is an adaptation. Our definition emphasises cooperation because we are interested in cases where individuals have been selected to work together, and so the actual dividing of tasks between individuals is an adaptation (see Supplementary Information S1 \& S2). It is the 'working together' that makes division of labour especially interesting from an evolutionary perspective, because it implies a cooperative adaptation across multiple individuals, to the benefit of those individuals. Van Gestel et al. ${ }^{7}$ have also emphasised the importance of division of labour being cooperative.

Our second and third conditions distinguish division of labour from cases where phenotypic variation has arisen as a by-product of otherwise self-interested traits, such as diversification to exploit different niches, or when one phenotype evolves to exploit another. We focus on inclusive fitness because it is our most general description of Darwinian fitness - natural selection favours traits that lead to an increase in inclusive fitness ${ }^{15-17}$ (Box 2). Consequently if we are interested in whether a social trait, such as division of labour, can be favoured, we examine the inclusive fitness consequences. Our definition makes no claim as to whether a population must be clonal, although we will discuss below how this can influence whether and what form of division is favoured.

Examples. When the bacterium Salmonella enterica subsp. enterica serovar imurium infects its vertebrate hosts, there is a division of labour between cells that stay in the gut lumen to reproduce, and cells that invade the gut tissue and express the type three secretion system, tts -1 , triggering an inflammatory response which eliminates 
competing bacteria from different species ${ }^{18,19}$ (FIG. 1A). The cells that enter the gut cooperative behaviour, which is costly to the invading cells but benefits the cells that remained in the gut lumen (FIG. 1B; see Supplementary Information S3). Another example of altruistic division of labour is provided by the fruiting bodies of slime moulds such as Dictyostelium discoideum ${ }^{20}$, where non-viable stalk cells hold up and help disperse the viable spore cells. Spore cells also occur in the fruiting bodies of Myxococcus xanthus ${ }^{21}$. These examples are analogous to that between germ and soma cells in multicellular species ${ }^{10}$.

Not division of labour. Our definition excludes a number of examples of phenotypic variation which are not cooperative, and hence not division of labour. When the bacterium Pseudomonas fluorescens is kept in liquid cultures, mutation leads to a range of different phenotypes, termed 'smooth', 'wrinkly spreader' and 'fuzzy spreader' ${ }^{22}$. This diversification does not represent an adaptive division of labour, it represents different lineages specialising to exploit different niches. Smooth inhabits the liquid phase, wrinkly spreader forms a mat at the air-broth interface, and fuzzy inhabits the less aerobic bottom of the broth. A variety of phenotypes also evolve in $P$. fluorescens colonies growing on agar ${ }^{23}$.

Another class of examples is provided by cases where cells can be divided between different lineages, where some perform cooperative behaviour and others do not perform, or perform less, of the cooperative behaviour. For example, Pseudomonas aeruginosa cells produce and excretes siderophores which scavenge for iron. The benefits of iron scavenging are shared between the local cells, and so this is a cooperative behaviour ${ }^{24}$. However, lineages evolve, both in laboratory broth cultures and in the lungs of humans with cystic fibrosis, that produce less or no siderophores ${ }^{25,26}$. These lineages appear to act as cheats, which exploit the siderophores produced by other cells ${ }^{8}$. In this example, the adaptation is the ability of cheaters (which produce less siderophores) to exploit co-operators (which produce more siderophores); this provides cheats with a selfish fitness benefit but decreases the fitness of the co-operators, and therefore does not constitute a division of labour. An analogous example of cheating is lineages that do not produce or respond to quorum sensing molecules ${ }^{27,28}$.

These examples illustrate that division of labour requires more than the production of a novel 'joint' phenotype or an ability to do something that wouldn't be possible without phenotypic diversity. For example, the different morphological variants of $P$. fluorescens can exploit the liquid media of a beaker in a way that would not be possible with a single phenotype ${ }^{22}$. It is not that these other types of phenotypic diversity are less interesting, just that they involve different problems than division of labour and result from different selection pressures. For example, cheats arise from conflict not cooperation, and do not require the efficiency benefits that we discuss below.

How to demonstrate division of labour? In order to demonstrate division of labour, two things need to be done. First, it must be shown that there is phenotypic variation, with different individuals specialising to perform different tasks. Second, it must be shown that this division is cooperative, providing a fitness benefit to the cells involved.

Considering the $S$. Typhimurium example discussed above, there is clearly phenotypic variation (some individuals express the $t t s s-1$, whereas other do not) ${ }^{18}$, but 
is it cooperative? One way to test whether microbial traits are cooperative is to grow 152 strains that do and do not perform the trait in both monocultures and mixed cultures ${ }^{29}$. Genetic manipulations allow the production of such strains. If the trait is cooperative and provides a benefit to others, then we would expect cells that perform the trait to grow best as monocultures (cooperators outperform cheats), whereas cells that do not perform the trait are predicted to grow best in mixed cultures (cheats can exploit cooperators; FIG. 1C) ${ }^{7,29}$.

Data from $S$. Typhimurium suggest that expression of ttss- 1 is an altruistically cooperative trait, and hence represents division of labour ${ }^{19,30}$. When grown in a monoculture, a mutant lineage that does not express ttss- 1 is avirulent and unable to spread within hosts (FIG. 1D). By contrast, when grown in a mixed culture with a wild type strain (that does express ttss-1), this mutant lineage is at an advantage and increases in frequency (FIG. 1D). Similar data exists for other cases, including the fruiting bodies of $D$. discoideum and $M$. xanthus, and the tubular mitochondrial morph in C. gattii $^{9,20,21}$.

It is necessary to carry out tests of whether a trait is cooperative in the environmental conditions under which that trait has evolved, or as near to it as possible $^{8}$. The costs and benefits of traits vary with environmental conditions, and so wrong conclusions can be made if tests are carried out in inappropriate environments ${ }^{31}$. With more complex traits and/or when labour is divided into more types, testing for cooperation can be harder. For example, in B. subtilis, two cell types are required to facilitate migration: cells that produce surfactin, a surfactant that reduces water surface tension, and cells that produce an extracellular polysaccharide matrix that glues cells together ${ }^{32}$.

\section{Why divide labour?}

Why would natural selection favour a division of labour, with different individuals performing different tasks, rather than just have everyone perform all the tasks? Consider a simple case with two tasks, A and B, where investment into these two activities must be traded off against each other, because time and energy spent on task A cannot be spent on task B. For example, task A might be reproduction and task B might be secreting a factor that causes an inflammatory response in the host, as in $S$. Typhimurium ${ }^{18,33}$. In these cases, a division of labour can be favoured when two conditions are met: (1) there is an efficiency benefit from different individuals performing different tasks (specialisation); (2) the reproductive interests of the different individuals are aligned, such that cooperation is favoured.

Efficiency benefits to division. Division of labour requires that there is some efficiency benefit to different individuals specialising in different tasks $7,10,34-38$. This means that when a cell puts a larger effort into a task, it obtains a larger return per unit invested. Assuming linear costs per unit invested into a task, this requires that the relationship between the proportion of resources that a cell allocates to a task and the fitness return is accelerating ${ }^{7,10,34,39}$ (FIG. 2A). An accelerating slope could arise if a task becomes more efficient as more effort is put into it, or if performing one of the tasks affects the ability to perform the other task (i.e., tasks A and B don't mix well, or are better done in different locations). By contrast, if the fitness returns on tasks are decelerating, with cells becoming less efficient the more they do of something, then natural selection favours all cells performing both tasks (FIG. 2A; see Supplementary Information S4). 
There is a lack of experimental work that explicitly tests for the kind of accelerating relationships illustrated in FIG. 2A, and this represents one of the largest gaps in our study of division of labour ${ }^{40}$. To date, arguments have tended to rely upon indirect extrapolations, rather than direct experimental tests. For example, consider the division between cells that photosynthesise and cells that fix nitrogen into ammonia (heterocycts) in some cyanobacteria species ${ }^{41}$. It has been argued that this division is favoured because nitrogenase, the enzyme that converts nitrogen gas to ammonia, is rapidly destroyed in the presence of oxygen, which is produced by photosynthesis $^{42}$. A direct experimental test of this hypothesis would require experimental manipulation of the extent to which nitrogen fixing and photosynthesis are divided between cells.

There are a number of other factors that could influence selection for division. For example, the relative returns from different tasks could vary with cell condition ${ }^{43}$. In some volvocine green algae, such as Volvox carteri, multicellular groups are composed of large germ cells that reproduce and smaller somatic cells that beat their flagella to keep the colony afloat ${ }^{44,45}$. This division appears to be favoured because large cells are more efficient at reproducing, which involves growth to a large cell size and then division, and small cells are better at both keeping the colony afloat and promoting diffusion of nutrients across the colony wall ${ }^{35,46,47}$. Another possibility is that cells in worse condition, such as smaller size or starved, could preferentially become the altruistic helper cells, rather than reproduce, if they have less to lose by not reproducing.

A major research aim is to explain why some species divide labour whereas other similar species do not? For example, it seems reasonable that the interaction between nitrogen fixing and photosynthesis could lead to the accelerating relationship in FIG. 2A, and hence lead to division of labour in cyanobacteria species such as Anabaena cylindrica. But that raises the problem of how do we explain other cyanobacteria, where this division of labour has not been favoured, such as Trichodesmium erythraeum ${ }^{41,42}$ ?

Alignment of interests. Division of labour requires that the fitness interests of different individuals are aligned. If not, the interaction between these individuals could be destabilised by selfish cheats, which can exploit the cooperative nature of division of labour. Examples of such cheats would be a $S$. Typhimurium strain that produced more cells that stayed in the gut lumen and never went into the gut tissue to express ttss-1, or a $D$. discoideum lineage that invested more into spore cells and less into stalk cells ${ }^{20,33,48}$.

One way for the interests of different individuals to be aligned is if they are genetically related ${ }^{15}$. This process, often termed kin selection, captures the idea that by helping a relative to reproduce, an individual is still indirectly passing on copies of its genes to the next generation (Box 2). Consequently, related cells can be favoured to work together via division of labour, in order to increase their genetic contribution to the next generation. Hamilton's rule ${ }^{15}$ (Box 2) predicts that altruistic traits (such as invading the gut tissue and expressing ttss- 1 in $S$. Typhimurium) will be favoured when both the relatedness between cells $(r)$ and the efficiency benefits $(B / C)$ are sufficiently high (FIG 2B). This illustrates that while division of labour is more likely with a higher relatedness, it does not require clonality $(r=1)$.

Four lines of evidence support the hypothesis that relatedness is important in favouring division of labour within species. First, anecdotally, many examples of division of labour occur in groups which are clonal $(r=1)$ or close to clonal. For 
example, cyanobacteria differentiate within clonal filaments ${ }^{41}$, and the average relatedness in fruiting bodies of the slime mould $D$. discoideum is $0.98^{49}$. Second, some species with division of labour exhibit kin discrimination during group formation. For example, individuals of the slime mould Dictyostelium purpereum preferentially form fruiting bodies with clone mates ${ }^{50}$. Third, the maintenance of cultures under low relatedness conditions led to the loss of the ability to form fruiting bodies in both $D$. discoideum and $M$. xanthus ${ }^{51}$, and a lower investment into somatic functions in the fungus Neurospora crassa ${ }^{52}$. Fourth, comparing across species, species with clonal group formation have greater division of labour, with both a higher likelihood of sterile cells and more cell types than species where group formation is non-clonal ${ }^{53}$ (FIG. 2C).

\section{What kind of division?}

In conditions where division of labour is favoured, we can then ask a range of more subtle questions. For example, what fraction of individuals should perform the different tasks? How many different cell types will labour be divided between? And which mechanisms are expected to give rise to division of labour?

How much division? Within the context of our theoretical example, where individuals perform either task A or task B, we can ask what is the evolutionarily stable strategy ${ }^{54}$ (ESS) fraction of individuals that should perform tasks $\mathrm{A}$ and $\mathrm{B}^{18,34,42}$. The ESS is an often used concept in evolutionary biology, to denote the strategy or behaviour that would win out over evolutionary time ${ }^{55}$. Put formally, it is the strategy which, if adopted by everyone in the population, cannot be invaded by any alternate strategy $y^{54}$.

The ESS will depend upon the shape of the fitness return curves (FIG. 2A) and the relatedness $(r)$ between interacting cells. One prediction is that, in clonal populations, the fitness of individuals will peak at the ESS fraction of A and B individuals, and then decrease as either A or B become more common ${ }^{18}$. For example, in the $S$. Typhimurium example, enough cells should express the type III secretion system to produce an inflammatory response, but any more than that is a waste ${ }^{18}$. Consistent with this prediction, mutants with either a lower or a higher fraction of cells expressing the type III secretion system showed a reduced fitness ${ }^{33}$.

Theory could be developed for specific cases to help explain both what the ESS is for certain situations and in what way should the ESS vary across populations or species. For example, in the volvocine green algae, the ratio of soma to germ cells increases with colony size ${ }^{44}$. It has been argued that this represents the shape of the fitness curve (FIG. 2A) changing with group size. Specifically, that as colony size increases, it becomes harder to keep the colony afloat and to transport nutrients, and so the ESS fraction of soma cells increases ${ }^{35,46}$.

We can ask questions about the variation in the fraction of cell types at a range of levels. For example, within species, why does the proportion of stalk cells in $D$. discoideum vary so widely across samples taken from the same location ${ }^{48,49}$ ? Across closely related species, why does the proportion of stalk cells vary across Dictyostelium species ${ }^{56}$ ? Across more distantly related species, why does the proportion of the altruistic reproductive phenotype vary from the approximately $20 \%$ of $D$. discoideum cells that become stalk, to the over $99 \%$ of Volvox cells that become soma ${ }^{20,57}$. ESS theory provides a tool for tackling these questions.

The ESS ratio of different types is also likely to vary with the relatedness between interacting individuals. However, there is a lack of both theory and empirical 
work examining how variation in relatedness would influence the ESS ratio of phenotypes ${ }^{18,53}$. Nonetheless, as relatedness gets lower, it will lead to a reduction in the kin selected benefit from helping others ${ }^{15}$, and so we suspect that a relatively general prediction is that a lower relatedness will lead to a lower proportion of individuals expressing altruistic phenotypes (FIG. 2B). Comparing across species, the percentage of sterile cells is twice as high in species with clonal groups, but this pattern is not statistically significant ${ }^{53}$. Although, there were only data for a small number of phylogenetically independent comparisons, and so this test had low statistical power.

How many types? Labour is sometimes divided into more than two types. For example, in cyanobacteria the number of cell types varies across species, up to at least four types ${ }^{41}$ (FIG. 1A). These cell types include photosynthetic cells, nitrogen fixing heterocysts, resting cells that are able to withstand environmental stress (akinetes) and motile dispersing filaments of cells (hormogonia).

We lack a formal theoretical framework for explaining variation in the number of cell types. A range of factors, are likely to be important, including ecological conditions, molecular mechanisms, relatedness within groups, and group size. Consistent with these possibilities, species which form clonal groups $(r=1)$ have more cell types than species where groups are not clonal $(r<1)$ (FIG. 2D), and species which form larger groups have more cell type ${ }^{53,58}$. A caveat here is that not all cell types represent division of labour, as phenotypic variation can arise for other reasons, such as bet hedging ${ }^{2,3}$.

Which way to divide? Given that there are many ways to produce variable phenotypes within a species (Box 1), what should we expect to occur in nature? Should we expect one mechanism to dominate or different mechanisms to mediate phenotypic variation in different species? And if mechanisms vary across species, does this represent adaptive variation, with different mechanisms being better suited to different situations, or is phenotypic variation just the result of noise created by historical artefacts? There has been no theoretical or empirical work addressing such questions, and so we make a number of tentative suggestions.

We suspect that it would require relatively restrictive conditions to maintain division of labour within species mediated by genetic differences. For example, most division of labour interactions occur within clonal lineages, where there will not be sufficient genetic differences. Furthermore, when multiple genetic lineages interact, this can reduce selection for cooperation ${ }^{59}$, and so whilst phenotypic variation can be maintained, it could be hard for such interactions to constitute a cooperative division of labour. For example, coexistence of producing and non-producing lineages, with traits such as invertase production in the yeast Saccharomyces cerevisiae, or siderophore production in the bacteria $P$. aeruginosa, appear to represent coexistence of cooperators and cheats, and not cooperative division of labour ${ }^{60,61}$. In cases where different genotypes could cooperate, there is the problem of how to keep them together over evolutionary time. Similar arguments would apply when considering epigenetic mechanisms ${ }^{62}$.

By contrast, phenotypic noise seems a robust way to produce division of labour. Noise can produce one or more phenotypes from a single genotype. The ratio of these genotypes could be selected upon, by selection on the underlying gene network, producing ratios that varied according to the ESS in the local environment ${ }^{2}$. A limitation with phenotypic noise is that it would work less well in small social 
groups. If the social group was a small number of cells, then stochasticity would lead to a chance that there were none, or almost none, of a certain phenotype in a social group, which could lead to a large fitness cost associated with maintaining phenotypic variation (FIG. 3). Consequently, we predict that phenotypic noise will be more likely to be the mechanism used when the social group is very large, as with expression of the ttss-1 system in Salmonella cells ${ }^{18,33}$.

Coordination between cells, with mechanisms such as signalling, provides a possible solution to the problem of stochasticity in small social groups. If cells interact and coordinate phenotypes at a local level, then this can ensure a precise and appropriate ratio of different phenotypes, even in small social groups, as occurs in cyanobacteria filaments $^{41}$ (FIG. 3). Given this advantage of signalling, why is it not used more frequently to control division of labour? Possibilities include that it could be relatively costly, or that it would be inaccurate in certain environments, such as when diffusion rates are high. In such cases, and when the problem of stochasticity is less important, such as with large social groups, phenotypic noise could provide a more efficient mechanism to divide labour.

\section{Outlook}

We have provided a definition of division of labour and discussed the implications associated with this definition. Is our definition useful? We have taken an evolutionary approach, focused on how individuals are adapted to their environments, and emphasised that division involves individuals working together. An alternative approach, focused on outcome rather than evolutionary adaptation, would be to define division of labour more loosely, as when phenotypic diversity allows more complex tasks to be carried out ${ }^{23}$. This alternative would include the examples we have excluded such as diversification, and one phenotype exploiting another.

The advantage of our stricter definition is that it brings together cases where the same problems arise, and where there is the potential for unifying understanding. For example, our stricter definition has illuminated the importance of shared interests and non-linear fitness returns in the evolution of division of labour (FIG. 2). By contrast, a looser definition would lump together traits that have evolved for very different reasons, and hence obscure underlying similarities. For example, the evolution of exploitation, or cheats that do not produce siderophores, does not require non-linear fitness returns, and is less likely to be favoured when individuals have shared interests. This illustrates the advantage of drawing a clear a distinction as possible between processes that arise for different reasons ${ }^{63}$.

Our definition resolves debates. Phenotypic heterogeneity between cells could arise in two broad ways. Either persistent specialisation, with some cells only carrying out task A, and other cells only carrying out task B, or via transient specialisation, with the same cell switching to do A and B at different times. In the social insect literature, it has been suggested that persistent specialisation is required for division of labour ${ }^{64}$. In contrast, by emphasising that what matters is cooperation, our definition clarifies that both persistent and transient specialisation could lead to division of labour.

To give another example of a debate, it has been suggested that the mutation of co-operators into non-cooperative cheats played a pivotal role in the evolution of the division between reproductive (germ) and helper (soma) cells, and hence the evolution of complex multicellularity ${ }^{65,66}$. If, however, we consider this hypothesis from an evolutionary perspective, both theory and empirical data contradict it. Theory suggests that selection would act in the opposite direction, favouring cheats that are 
better able to exploit cooperators, and cooperators who are less exploitable ${ }^{8,11}$. The often in species with clonal group formation, where there is no selection for cheating $^{53}$ (FIG. 2C).

Based on the multiple examples that have been described in recent years, these are exciting times for the study of division of labour in microorganisms. Much remains to be done, both theoretically and empirically. In many cases, it remains to even be demonstrated if examples of phenotypic variation really represent division of labour. In cases where a division of labour is demonstrated, this raises a whole slew of further questions. Why is division favoured? How many different phenotypes, and what fraction should each be? What mechanism is used to generate phenotypic diversity and why? Can we explain variation across species, as well as specific cases? Can we apply the same concepts to explain division of labour between species (see Supplementary Information S6)? By answering these questions we can unify our understanding of division of labour, not only with mechanistically very different microbial examples, but also with examples from other taxa, including animal societies.

\section{Acknowledgements}

We thank Koos Boomsma, Alfonso Perez Escudero, Kevin Foster, Andy Gardner, Melanie Ghoul, Jeff Gore, Ashleigh Griffin, Robin May, Cláudio Nunes-Alves, Joan Strassmann, Daniel Unterweger \& Jordi van Gestel for very useful discussion. Martin Ackermann, Robin May, Rich Michod \& Jan-Willem Veening provided photographs.

\section{Text boxes}

\section{Box 1. Mechanisms of phenotypic variation.}

There are at least six possible mechanisms for generating phenotypic variation within a species ${ }^{2,67}$. There can be overlap between these mechanisms.

Genetics. There could be genetic differences, with different genotypes leading to different phenotypes, via standing genetic variation or mutation ${ }^{23}$.

Epigenetics. Different phenotypes could be maintained by epigenetic inheritance, such as DNA methylation, leading to a correlation in phenotype across generation ${ }^{68}$. Noise. If noise in the biochemistry of the cell is coupled with a gene network that amplifies small differences, then this can lead to phenotypic variation ${ }^{2,67}$. Phenotypic noise is the basis for examples such as that between cells which do and don't express ttss-1 in S. Typhimurium, or exoproteases in Bacillus subtilis ${ }^{5,18}$.

Signalling. In cyanobacteria, such as Anabaena species, signaling peptides are exported from cells to their neighbours, to control which cells develop into nitrogen fixing heterocysts ${ }^{41}$. This produces a regular pattern of heterocysts every relatively fixed number of vegetative cells along the filamentous colony. This number can vary from $\approx 4-15$ cells, depending upon the species.

Environment. Variation can be generated by environmental cues. For example, in cyanobacteria, in addition to the role of signaling, nitrogen stress can lead to a higher proportion of cells developing into heterocysts ${ }^{41}$.

Condition dependence. Variation can be generated by differences in cell condition. In $V$. carteri, a series of asymmetric cell divisions, during early embryonic development, lead to small and large cells, which develop into soma and germ respectively ${ }^{69,70}$. This process involves a gene that ancestral ancestors used to reduce reproduction in stressful conditions, being co-opted to produce a non-reproductive 
phenotype $^{69,71,72}$. Variation in condition can also be environment dependent, for example lower condition starving cells might be more likely to become a nonreproductive altruist.

Complementary or competing approaches? It is useful to distinguish between mechanistic and evolutionary approaches to studying traits such as phenotypic heterogeneity ${ }^{73}$. The mechanistic (proximate) approach is to ask questions about how traits are controlled, such as what are the molecular or genetic mechanisms that control a particular trait (how questions). The evolutionary (ultimate) approach is to ask questions about the fitness consequences of that trait, and why it has been favoured by natural selection (why questions).

The majority of previous work on phenotypic heterogeneity has been mechanistic - our aim in this paper is to ask evolutionary questions ${ }^{2,3,6,7,41,67}$. These two approaches are complementary and not competing. Mechanistic answers cannot be given for evolutionary questions and vice versa, but an understanding from one perspective can aid the other perspective ${ }^{29}$. For example, an evolutionary approach can suggest when we might find different mechanisms in different species, whereas a mechanistic understating of what factors stimulate phenotypic heterogeneity can help us understand why that heterogeneity is favoured.

\section{Box 2. Natural selection and adaptation}

Natural selection favours genes that are better at transmitting to the next generation ${ }^{74}$. However, researchers often talk about natural selection acting on individual behaviour, leading to individuals that maximise their fitness. The formal justification for this thinking at the individual level is that genes which increase fitness will accumulate, and so natural selection, via gene dynamics, will lead to organisms that behave as if they are trying to maximise their fitness ${ }^{74,75}$. Thus, the gene and individual approaches are not competing, they are flip sides of the same coin - gene dynamics leads to individual fitness maximisation.

Genes can influence their transmission to the next generation, not only by influencing the reproductive success of the individual that they are in, but also by influencing the reproductive success of other individuals carrying that gene. Hamilton ${ }^{15}$ showed that natural selection will lead to individuals that behave as if they are maximising not their personal reproductive success, but what he called inclusive fitness. Inclusive fitness is the sum of fitness obtained directly, through reproduction, and indirectly through influencing the reproduction of relatives. Indirect fitness must be weighted according to relatedness, which is a statistical measure of the genetic similarity between individuals.

Hamilton's theory is often discussed in terms of kin selection, and Hamilton's rule $^{15}$. Hamilton's rule shows that an altruistic trait, such as becoming a sterile stalk cell in a fruiting body, will be favoured when $r B-C>0$; where $C$ is the fitness cost of performing the trait, $B$ is the fitness benefit to other individuals, and $r$ is the genetic relatedness to the individuals receiving the benefit. The most common ways for interacting cells to be related are by either limited dispersal keeping relatives together, or kin discrimination mechanisms which allow individuals to preferentially interact with relatives ${ }^{15}$.

Division of labour is often discussed as benefitting the population or community, with cells behaving analogous to a multicellular organism. It is useful to ask whether this is justified. More formally, we can ask when would gene dynamics lead to individuals that are trying to maximise their group or population fitness? This requires extremely restrictive conditions, where there is effectively no conflict within 
groups, such as in clonal populations of cells ${ }^{63,76,77}$. The cells that make up complex multicellular organisms, such as humans, fit this criterion, but populations or communities of microorganisms might not. Consequently, thinking about adaptations such as division of labour at the group or population level is not formally justified and can lead to errors with microorganisms.

\section{FIGURE LEGENDS}

\section{Fig. 1. Division of labour.}

(a) Potential examples of division of labour. The photos show phenotypic variation in: (i) Volvox carteri (large germ cells, and small soma cells; from ${ }^{78}$ ); (ii) Bacillus subtilis (cells which are (green) and are not (grey) producing proteases; from ${ }^{5}$ ); (iii) $S$. Typhimurium (cells which are (green) and are not (grey) expressing the type three secretion system; from ${ }^{30}$ ); and (iv) Cryptococcus gatti (the cell on its own has tubularised its mitochondria (the yellow), whereas the others have not; Simon Johnston \& Robin May). (b) Division of labour can involve either cooperation in one direction where individuals of one phenotype help another phenotype (usually altruistically), or cooperation in both directions, where individuals of each phenotype cooperate with each other (possibly to their mutual benefit; see Supplementary Information S3). (c) One way to demonstrate division of labour is to grow strains that do and do not perform the putatively cooperative trait, in both monocultures and mixed cultures. We consider: a cooperative strain, with division of labour, where cells develop into both the altruistic helping phenotype (blue cells) and the reproducing phenotype (green cells); and a cheat strain, where all cells develop into the reproducing phenotype (black cells). In monocultures, the cooperative strain, with division of labour, grows at a greater rate. In contrast, in mixed cultures, the cheats grow at a faster rate. This prediction arises because relatively non-cooperative 'cheats' can exploit the benefits provided by the altruistic helping phenotype, when they are grown with the cooperative strain. (d) An example of this predicted pattern is provided by data from two strains of $S$. Typhimurium - the wild type with a relatively normal division of labour, and an ahilD mutant, that produces less of the phenotype that triggers the type III secretion system. The wild type has a relatively higher fitness when grown in monoculture, as measured by cells per gram of faeces, but a relatively lower fitness when grown in a mixed culture. Data from Diard et al. ${ }^{19}$.

\section{Fig 2. Why Divide Labour?}

(a) The relationship between the proportion of resources that a cell invests into a task $\mathrm{A}$ and the fitness return from that task. We assume that a cell invests proportion of resources $X$ into task $\mathrm{A}$, and the remaining proportion $1-X$ into task B. Division of labour can be favoured when the returns from investment are accelerating. (b) The hypothetical relationship between the proportion or likelihood of individuals specializing in task A, and the relatedness $(r)$ between interacting individuals in a social group. Division of labour is only favoured above a threshold value of relatedness $\left(r_{c}\right)$. (c,d) Across the tree of life, a higher relatedness within multicellular groups is correlated with both: (c) a higher likelihood of sterile cells; and (d) more cell types. The relatedness comparison is between groups which form clonally $(r=1)$ and groups which form non-clonally $(r<1)$. Data from Fisher et al. ${ }^{53}$.

\section{Fig. 3. How to divide?}


549 Consider a population that is composed of altruistic cells (blue) and reproductive cells 550 (green). Determination of phenotype occurs via phenotypic noise or via a coordinated 551 mechanism such as between cell signalling. The number of cells that interact in the social group can be either large or small. In large groups, both phenotypic noise and coordinated division lead to groups with the appropriate number of altruists.

554 Consequently, both mechanisms could be favoured to divide labour. If coordinated 555 division is more costly, for example because it requires the metabolic cost of 556 producing a signal, then phenotypic noise will be the favoured mechanism. In small 557 social groups, the stochastic nature of phenotypic noise will mean that there is an 558 appreciable chance that the group contains no altruists, and so have low fitness (these 559 groups are circled in red). In contrast, coordinated division provides a mechanism to 560 ensure that all groups contain altruists. Consequently, phenotypic noise is less likely 561 to be favoured as a mechanism to divide labour in species where the number of 562 interacting cells is small. 
1. Lewis, K. Persister cells, dormancy and infectious disease. Nat Rev Micro 5, 48-56 (2006).

2. Veening, J.-W., Smits, W. K. \& Kuipers, O. P. Bistability, Epigenetics, and Bet-Hedging in Bacteria. Annu. Rev. Microbiol. 62, 193-210 (2008).

3. Ackermann, M. A functional perspective on phenotypic heterogeneity in microorganisms. Nat Rev Micro 13, 497-508 (2015).

4. Balaban, N. Q., Merrin, J., Chait, R., Kowalik, L. \& Leibler, S. Bacterial persistence as a phenotypic switch. Science 305, 1622-1625 (2004).

5. Veening, J.-W. et al. Transient heterogeneity in extracellular protease production by Bacillus subtilis. Molecular Systems Biology 4, 1-15 (2008).

6. Claessen, D., Rozen, D. E., Kuipers, O. P., Søgaard-Andersen, L. \& van

Wezel, G. P. Bacterial solutions to multicellularity: a tale of biofilms, filaments and fruiting bodies. Nat Rev Micro 12, 115-124 (2014).

7. van Gestel, J., Vlamakis, H. \& Kolter, R. in Microbial Biofilms, 2nd Edition 67-97 (American Society of Microbiology, 2015). doi:10.1128/microbiolspec.MB-0002-2014

8. Ghoul, M., Griffin, A. S. \& West, S. A. Toward an evolutionary definition of cheating. Evolution 68, 318-331 (2014).

9. Voelz, K. et al. 'Division of labour'in response to host oxidative burst drives a fatal Cryptococcus gattii outbreak. Nature Communications 5, 5194 (2014).

10. Maynard Smith, J. \& Szathmáry, E. The Major Transitions in Evolution. (Oxford University Press, 1995).

11. Bourke, A. F. G. Principles of Social Evolution. (OUP Oxford, 2011).

12. West, S. A., Fisher, R. M., Gardner, A. \& Kiers, E. T. Major evolutionary transitions in individuality. Proceedings of the National Academy of Sciences 112, 10112-10119 (2015).

13. Crespi, B. J. \& Yanega, D. The definition of eusociality. Behavioral Ecology 6, 109-115 (1995).

14. West, S. A., Griffin, A. S. \& Gardner, A. Social semantics: altruism, cooperation, mutualism, strong reciprocity and group selection. J. Evol. Biol. 20, 415-432 (2007).

15. Hamilton, W. D. The genetical evolution of social behaviour. I. Journal of Theoretical Biology 7, 1-16 (1964).

16. Grafen, A. Optimization of inclusive fitness. Journal of Theoretical Biology 238, 541-563 (2006).

17. West, S. A. \& Gardner, A. Adaptation and Inclusive Fitness Review. Current Biology 23, R577-R584 (2013).

18. Ackermann, M. et al. Self-destructive cooperation mediated by phenotypic noise. Nature 454, 987-990 (2008).

19. Diard, M. et al. Stabilization of cooperative virulence by the expression of an avirulent phenotype. Nature 494, 353-356 (2013).

20. Strassmann, J. E., Zhu, Y. \& Queller, D. C. Altruism and social cheating in the social amoeba Dictyostelium discoideum. Nature 408, 965-967 (2000).

21. Velicer, G. J., Kroos, L. \& Lenski, R. E. Developmental cheating in the social bacterium Myxococcus xanthus. Nature 404, 598-601 (2000).

22. Rainey, P. B. \& Travisano, M. Adaptive radiation in a heterogeneous environment. Nature 394, 69-72 (1998).

23. Kim, W., Levy, S. B. \& Foster, K. R. Rapid radiation in bacteria leads to a 
and early radiation of multicellular volvocine algae. Proceedings of the National Academy of Sciences 106, 3254-3258 (2009).

46. Solari, C. A., Kessler, J. O. \& Michod, R. E. A Hydrodynamics Approach to the Evolution of Multicellularity: Flagellar Motility and Germ- Soma Differentiation in Volvocalean Green Algae. Am Nat 167, 537-554 (2006).

47. Solari, C. A., Ganguly, S., Kessler, J. O., Michod, R. E. \& Goldstein, R. E. Multicellularity and the functional interdependence of motility and molecular transport. Proceedings of the National Academy of Sciences 103, 1353-1358 (2006).

48. Wolf, J. B. et al. Fitness Trade-offs Result in the Illusion of Social Success. Current Biology 25, 1086-1090 (2015).

49. Gilbert, O. M., Foster, K. R., Mehdiabadi, N. J., Strassmann, J. E. \& Queller, D. C. High relatedness maintains multicellular cooperation in a social amoeba by controlling cheater mutants. Proceedings of the National Academy of Sciences 104, 8913-8917 (2007).

50. Mehdiabadi, N. J. et al. Kin preference in a social microbe. Nature 442, 881882 (2006).

51. Kuzdzal-Fick, J. J., Queller, D. C., Fox, S. A. \& Strassmann, J. E. High relatedness is necessary and sufficient to maintain multicellularity in Dictyostelium. Science 334, 1548-1551 (2011).

52. Bastiaans, E., Debets, A. J. M. \& Aanen, D. K. Experimental evolution reveals that high relatedness protects multicellular cooperation from cheaters. Nature Communications 7, 1-10 (2016).

53. Fisher, R. M., Cornwallis, C. K. \& West, S. A. Group Formation, Relatedness, and the Evolution of Multicellularity. Current Biology 23, 1120 1125 (2013).

54. Maynard Smith, J. \& Price, G. R. The Logic of Animal Conflict. Nature 246, 15-18 (1973).

55. Dawkins, R. The Selfish Gene. (Oxford University Press, 1976).

56. Bonner, J. T. Cellular slime molds. (Princeton University Press, 1967).

57. Shelton, D. E., Desnitskiy, A. G. \& Michod, R. E. Distributions of reproductive and somatic cell numbers in diverse Volvox (Chlorophyta) species. Evolutionary Ecology Research 14, 707-727 (2012).

58. Bell, G. \& Mooers, A. O. Size and complexity among multicellular organisms. Biological Journal of the Linnean Society 60, 345-363 (1997).

59. Frank, S. A. Host-symbiont conflict over the mixing of symbiotic lineages. in (1996).

60. Gore, J., Youk, H. \& van Oudenaarden, A. Snowdrift game dynamics and facultative cheating in yeast. Nature 459, 253-256 (2009).

61. Ross-Gillespie, A., Gardner, A., West, S. A. \& Griffin, A. S. Frequency Dependence and Cooperation: Theory and a Test with Bacteria. Am Nat 170, 331-342 (2007).

62. Haig, D. Weismann Rules! OK? Epigenetics and the Lamarckian temptation. Biol Philos 22, 415-428 (2006).

63. Maynard Smith, J. Group selection. (1976).

64. Gordon, D. M. From division of labor to the collective behavior of social insects. Behav Ecol Sociobiol 1-8 (2015). doi:10.1007/s00265-015-2045-3

65. Rainey, P. B. \& Kerr, B. Cheats as first propagules: A new hypothesis for the evolution of individuality during the transition from single cells to multicellularity. Bioessays 32, 872-880 (2010). 
66. Hammerschmidt, K., Rose, C. J., Kerr, B. \& Rainey, P. B. Life cycles, fitness decoupling and the evolution of multicellularity. Nature 515, 75-79 (2015).

67. Davidson, C. J. \& Surette, M. G. Individuality in Bacteria. Annu. Rev. Genet. 42, 253-268 (2008).

68. Veening, J.-W. et al. Bet-hedging and epigenetic inheritance in bacterial cell development. Proceedings of the National Academy of Sciences 105, 43934398 (2008).

69. Kirk, D. L. Asymmetric division, cell size and germ-soma specification in Volvox. Seminars in Developmental Biology 6, 369-379 (1995).

70. Schmitt, R. Differentiation of germinal and somatic cells in Volvox carteri. Current Opinion in Microbiology 6, 608-613 (2003).

71. Nedelcu, A. M. \& Michod, R. E. The evolutionary origin of an altruistic gene. Molecular Biology and Evolution 23, 1460-1464 (2006).

72. Hanschen, E. R., Ferris, P. J. \& Michod, R. E. Early evolution of the genetic basis for soma in the Volvocaceae. Evolution 68, 2014-2025 (2014).

73. Tinbergen, N. On aims and methods of Ethology. Zeitschrift für

Tierpsychologie 20, 410-433 (1963).

74. Fisher, R. A. The Genetical Theory of Natural Selection. (Clarendon, 1930).

75. Grafen, A. The formal Darwinism project: a mid-term report. J. Evol. Biol. 20, 1243-1254 (2007).

76. Leigh, E. G., Jr. When does the good of the group override the advantage of the individual? in (1983).

77. Gardner, A. \& Grafen, A. Capturing the superorganism: a formal theory of group adaptation. J. Evol. Biol. 22, 659-671 (2009).

78. Shelton, D. E. \& Michod, R. E. Philosophical foundations for the hierarchy of life. Biol Philos 25, 391-403 (2009). 